\title{
ABSTRACTION AND UNIVERSALS IN THEORIZING ABOUT LANGUAGE EVOLUTION
}

\author{
RICHARD SHILLCOCK \\ rcs@inf.ed.ac.uk
}

Philosophy, Psychology \& Language Sciences, The University of Edinburgh, UK

\begin{abstract}
Philosophical problems in the study of language evolution are introduced in connection with a range of different categories: totality, abstraction, universal, development, materiality. The approach which emerges contrasts with conventional theorizing in the field. The concept of 'concrete universal' is applied to reveal a continuity between the earliest necessary stages of language evolution and fully-developed contemporary language-use.
\end{abstract}

\section{Philosophical problems in the language evolution field}

Theorizing and experimentation come with often unexplored philosophical assumptions. I will address issues concerning the category of universal and associated categories, from the perspective of an explicit theory of knowledge. I will then develop a claim concerning continuity between the earliest stages in the evolution of language and fully-developed language use.

\subsection{The totality}

The scientific enterprise begins with the totality of the domain under investigation. For language evolution, it is the totality of our human ancestors collectively scraping an existence in the context of nature and culture, where the latter refers to habit, artefact, and ritual. (See MacWhinney, 2005, for a summary of some of the many factors that may have been important in language evolution.)

Critically, for the theory of knowledge employed here, the enterprise also ends with this same totality. As we will see, we will analyse this totality so as to identify component parts and characteristic mechanisms, but the exercise will end with us taking the 'return trip' away from partial views of the totality and finishing with the reconstituted totality. The scientific enterprise does not end with an analogy 
between the totality and a representation couched in formal logic or in abstract mathematical terms. It ends back at the totality of the domain.

A second way in which cognitive scientists conventionally end up with partial views of the domain of language processing is that subdomains are considered in isolation: syllabicity, lexical stress, lexis, syntax, semantics, prosody, morphology, phonology and so on. The case of syntax is illustrative. The central phenomena - the 'fat' part of the distribution of sentence structures, the most frequent structures - are easily characterized, the challenge being to understand the peripheral phenomena, the 'long tail' of the distribution of sentence structures consisting often of only single instances in a large corpus (Steedman, pers. comm.). These rare items are caused by idiosyncratic interaction with the other subdomains. Hence the attention of formal linguists turns to such interaction-to morphosyntax, morphophonology and so on. The point I make here is that for abstract systems to interact there requires to be some form of mediation. The more abstract systems that interact, the likelier it is that the mediator will be something material, something real we can observe and manipulate. A material mediator is richer in potential 'points of contact' and is maximally applicable to new forms of interaction. (We return below to this issue.)

A third way in which cognitive science approaches to language processing remain at an abstract level is the conventional goal-implicit in the typical approach to universals - of generalizing across all languages, of characterizing Language with a capital $\mathrm{L}$. The overall tendency is for 'explanation' to apply to Language and 'description' to apply to specific languages. The approach pursued here is concerned with material explanation. In the second half of the paper we address the case of English. This move is not due to the convenience that more psycholinguistic research exists for English than for any other language. Rather it is a recognition that the approach should be applied to material mechanisms in real and typical cases. Any real language is a suitable domain of study. The best explanation for the development of English cannot be disproved or improved by data from an unconnected language. We return below to the question of other languages.

\subsection{Abstraction}

To make the initial totality tractable, we need to abstract from it. The type of abstraction required is an 'Aristotelian abstraction', in which we take away (in the mind of the analyst) aspects of the totality ( $c f$. Jones, 2005). This is fundamentally different from 'Galilean abstraction' and the example of the frictionless plane that is typically cited (cf. Weisberg, 2007). This latter approach will mislead us. First, 
because rather than taking something away from the domain, it is truer to say that Galilean abstraction replaces something; a plane that involves real friction has been replaced by an abstract plane that stands for that which is similar across all planes of varying friction. Second, experience shows that researchers that employ Galilean abstraction frequently do not cash in the promise to return to the complete domain, preferring instead to stay with the abstract view of the domain.

\subsection{A universal}

What are we expecting to find by successively taking away material aspects of the domain? Our goal is to identify the simplest entity that itself simultaneously exhibits and explains the behaviours we see in the domain.

Vygotsky (1934/86) termed such an entity the 'unit of analysis'. His example was the investigation of the fluid properties of water. The relevant unit is the water molecule. Its constituent hydrogen and oxygen atoms are too small to exhibit the relevant behaviours — van der Waals force, etc. - that lead us to an understanding of the fluidity of water.

Such an entity is a universal. It unifies many things - the definition of a universal. It participates as itself in the relevant domain. We required to discover it in the domain. It mediates everything else in the domain, in which it is pervasive. It is a material entity - it is not going to be 'disproved' or wished away by new data.

Such a universal is different in all these respects from the type of universal that cognitive scientists are used to employing. Take 'verb'. This is an entity that needed to be created, not discovered. The category 'verb' does not participate as itself in the domain (although we may initially think so, because we tend to turn formal linguistic categories into processing categories). Verb is certainly a useful category, though. It captures generalizations about the domain, in the form of ordered relations between itself and other such abstract universals (syntax being the paradigm example of such relationships). But this is the limit of the category 'verb', combined with the fact that it may be eclipsed by new data (such as the weakness of the formal distinction between nouns and verbs in the Salish languages; Jelinek, 1995).

This less conventional universal has a long philosophical history (Shillcock, 2014) in which it is termed the concrete universal, signaling its role in connecting other entities. 


\subsection{Explanation}

In this materialist theory of knowledge, explanation consists in having identified the concrete universal within the domain by a process of successively abstracting away the peripheral aspects of the domain and in then being able to reverse the process by successively taking this or that aspect of context and demonstrating the necessary implications it has for the role of the concrete universal within the domain, until the totality of the domain has been recreated.

If at any point in the movement between the concrete universal and the totality, we can say that adding this aspect of context causes this to happen, and subtracting this other aspect of context causes this other thing to happen, then we have achieved an explanation of how the domain works. We will have understood the 'logic' of the domain - not in the sense of some abstract formalism, but in terms of the material role of the concrete universal.

Another example. The stem cell in the domain of human anatomy graphically represents Vygotsky's notion of the unit of analysis or cell-form of a domain. The stem cell participates as itself in the domain and exhibits all of the basic characteristics of the other cells of the body. The stem cell is pervasive, it mediates the life of every other cell in that all other cells are derived from stem cells. If we can specify every aspect of the contexts that necessarily cause a stem cell to turn into a neuron, a skin cell, a lung cell, and so on, and we can characterize the effects on the stem cell at each point in the emergence of the organs of the body, then we will have explained human anatomy.

\subsection{The genetic approach to studying language}

The approach outlined above is essentially a genetic approach ( $c f$. Vygotsky, 1934/1986). A domain is understood by exploring how it came into being. In this sense, the study of the development of language (phylogenetic and ontogenetic) is the best way to understand language.

At the same time, the current necessity of beginning with the developed form of language (as opposed to having access to some still-existing earlier stage) is actually a privileged viewpoint, in that we can identify potentially insignificant aspects of the possible beginnings of language that go on to become crucial in the developed form. Similarly, we can put into perspective potentially important aspects that have little role in the fully developed form. 


\subsection{Materiality of a concrete universal}

It is the material nature of the concrete universal that goes most against the grain of conventional cognitive science. The credo of 'Marr's levels' (Marr, 1982) has as its highest level the computational specification of the problem- the laws of arithmetic, in the typical example. Marr abstracted from the totality of the processing and did not then perform the 'return journey' to explain how any essence of the processing might emerge in the first place and develop into the observed role in the full context.

In the present approach to explanation, we require both individuality and generality. We require to know exactly what is going on throughout the domain of study. Nothing can be absolutely individual or absolutely general. It is in the dialectic between these two goals that the concrete universal emerges - an entity as detailed as possible and with as pervasive a mediating role as possible. These requirements necessitate a material entity as the concrete universal in the domain.

\section{Applying the analysis}

Below, the approach sketched in 1 is applied to the evolutionary emergence of language. There is necessarily a continuum of development with the processing of current English. How can our understanding of the latter inform our exploration of language evolution?

The first move we must make is to define the domains with which we are concerned. Depending on the content of the domain, a different concrete universal will apply; the 'essence' of what goes on in the domain will be different. We can consider three domains: 'general human cognition', 'communication', 'contemporary English'.

\subsection{The domain of general human cognition}

The theoretical positions of this paper suggest that hemisphericity is central to the emergence of general human cognition (Shillcock, Thomas, \& Bailes, submitted). Hemisphericity is the relative encapsulation, differentiation and autonomy of the cerebral hemispheres; the degree of hemisphericity distinguishes the human brain from the even our closest primate relatives. The material requirement of the hemispheres to coordinate themselves is the relevant concrete universal. This coordination necessitates each hemisphere modelling the other in the context of body and world, effectively 'parsing the world' and thereby generating tools for cognition. 'Predictive Processing' (Friston, 2005; Clark, 2015) between the hemispheres is seen as playing an important role in this mutual modelling, but 
each hemisphere effectively uses the other hemisphere as a tool. We return to the role of the hemispheres below.

\subsection{The domain of communication}

What is the concrete universal in the domain of communication? Perhaps the best candidate for the 'cell-form' in this domain is the addresser materially re-orienting the addressee between old and new information: "you already know this" vs. "this is news". This distinction is the very basis of communication. The use of 'orienting' here recalls the orienting response (Sokolov, Spinks, Näätänen, \& Lyytinen, 2002) to an external stimulus and a similar response to an 'internal stimulus'.

Language and cognition are heavily-tilled fields and we can expect to encounter familiar distinctions; in this case, Sperber and Wilson's (1995) 'relevance'. What is new here is situating the distinctions within the deeper particular philosophical and psycholinguistic contexts.

We should expect to be able to identify some material orienting activity by the addresser. Fundamentally, the addresser uses the addressee as a tool to achieve some purpose, as in physically steering them to participate in joint activity. Physical contact, non-contact gesture, and speech sounds are all equivalent in the sense that they reorient the addressee between old and new information. They naturally differ in their directness and sophistication, spoken language being the most developed form of re-orienting the addressee.

If we limit our considerations to speech, then the minimal vocal gesture is that produced by the vocal apparatus approximating a simple tube-a spectrally diffuse schwa-like sound ( $c f$. Harris, 1995). We can identify such a minimal reorienting activity with the contemporary cross-linguistic vocal gesture identified by Dingemanse, Torreira and Enfield (2013) as 'other-initiated repair'. In this joint activity, the interlocutors negotiate the relationship between old and new information.

\subsection{The domain of contemporary English}

We have identified a material universal - the activity of producing a schwa-like sound-as playing the role of re-orienting the addressee between old and new information. The critical move we now make is to continue exploring the role of this concrete universal beyond the simple routine of repair initiation and repair solution. 
Vocal activity approximating a schwa sound accounts for some $10 \%$ of conversational English (Fry, 1947). It fits the bill for a concrete universal for contemporary English by virtue of being pervasive within the domain.

Schwa-like activity in spoken English has a still stronger claim to be the relevant concrete universal. It mediates every other entity in the domain. The speech activity that is conventionally labelled schwa has an idiosyncratic relationship with each of the formal subdomains explored in contemporary spoken English, from articulatory phonetics, through phonology, lexical stress, syllabic structure, the functor/contentive distinction, syntax (by way of projection from functional heads), and even word meaning.

Consider just one example of schwa-activity from the above partial list. Schwa-activity is a reliable cross-linguistic indicator of functorhood (Shi, Morgan, \& Allopenna, 1998). A critical development in syntactic theory occurred with the identification of the functor as the syntactic head of the phrase as opposed to the noun. This change was very productive in revealing systematicity in syntax. It is one instance in which schwa-activity constitutes the material bridgehead into a formal subsystem of language (syntax).

Schwa-activity constitutes a similar material bridgehead into the other formal subsystems of contemporary English. It is contained in each subsystem, constrained in very specific ways, and is a material way in which those subsystems articulate one with another.

The claim here is emphatically not that developed linguistic behaviour reduces to schwa-activity. It is that schwa-activity in its primitive form of negotiating the relationship between old and new information is the basic cell-form that can characterize and explain more fully developed linguistic behaviour.

Thus, the simple form of schwa-activity may be elaborated phonologically (adding or subtracting behaviours corresponding to features) to provide the wherewithal to make more detailed linguistic references. It mediates subsystems such as syllabicity and lexical stress, which in turn provide more structure and expressive potential.

Critically, schwa-activity in contemporary English signals old information, a return to the shared world, a retreat from new information (see, e.g., Fowler \& Housum, 1987). It occurs in functors signalling known aspects of the discourse such as tense and number. It occurs in parts of words beyond the uniqueness point.

\subsection{The constitutive role of schwa-activity in processing}

The claim was rehearsed above that the necessity of hemispheric coordination was the concrete universal that generated distinctively human cognition. This 
occurs by mutual hemispheric modelling occurring partly by direct callosal interaction, partly by predictive processing, and partly by the tool-mediated interaction of the contralaterally-controlled manipulators.

The frequent occurrence of schwa-like activity in speech marshals the hemispherically-distributed processing preferences for prosody, phonology, different aspects of syntax, differently parameterized semantics, face processing, lexis and so on. It reinforces their mutual relationships.

This approach to a theory of knowledge thus takes us from the emergence of communication within the species, where the concrete universals suggested-the necessity of hemispheric communication and the orientation between old and new information - will be fundamental and it delivers us to the more idiosyncratic constraints of the cultural evolution of a specific language, where the suggested concrete universal-schwa-like activity-works for English, but where some analogous speech activity may be required for different languages.

\section{Conclusions}

We have introduced a universal, not conventionally recognized as such, but assumed by a materialist theory of knowledge - the concrete universal. We have applied this theoretical tool to the domains relevant to the early stages of language evolution - 'general human cognition' and 'communication'-and we have identified the material 'cell-form' of these domains-schwa-like activity. We have then crucially suggested a continuity between these early aspects of language evolution and the continuing development of linguistic activity and structure. Our domain of application has been contemporary English, but the prediction is that an analogous analysis can be made for any other real and typical language.

The approach we have followed firmly orients researchers towards the material richness of language behaviours and encourages them to interrogate their models - from box-and-arrow diagrams to computational implementations - as to the precise status of each entity in the model. Experience shows that the serious limitations of abstract universals are overlooked and that the merits of concrete universals are not appreciated.

The goal has been to explore the 'logic' by which fully-fledged linguistic activity works and to draw a line of material continuity between such fullydeveloped processing and the earliest stages of language evolution.

\section{References}

Clark, A. (2015). Surfing uncertainty: Prediction, action, and the embodied mind. Oxford University Press. 
Dingemanse, M., Torreira, F., \& Enfield, N. J. (2013). Is "Huh?" a Universal Word? Conversational Infrastructure and the Convergent Evolution of Linguistic Items. PloS one, 8(11), e78273.

Fowler, C. A., \& Housum, J. (1987). Talkers' signaling of "new" and "old" words in speech and listeners' perception and use of the distinction. Journal of Memory and Language, 26(5), 489-504.

Friston, K. (2005). A theory of cortical responses. Philosophical Transactions of the Royal Society of London B: Biological Sciences, 360(1456), 815-836.

Fry, D.B. (1947). The frequency of occurrence of speech sounds in Southern English. Archives Néerlandaises de Phonétique Expérimentale, 20, 103-106.

Harris, J. (2005). Vowel reduction as information loss. In P. Carr, J. Durand, \& C. J. Ewen (eds.), Headhood, Elements, Specification and Contrastivity, pp. 119-132. Amsterdam: Benjamins.

Jelinek, E. (1995) Quantification in Straits Salish. In: Quantification in natural languages, ed. E. Bach, E. Jelinek, A. Kratzer \& B. Partee, pp. 487-540. Kluwer.

Jones, M. (2005). Idealization and abstraction: A framework. In M. Jones and N. Cartwright (eds.), Idealization XII: Correcting the Model: Idealization and Abstraction in the Sciences (New York: Rodopi), 173-217.

MacWhinney, B. (2005). Language evolution and human development. In Bjorklund, D. and Pellegrini, A. (Eds.). Origins of the Social Mind: Evolutionary Psychology and Child Development, New York, Guildford Press.

Marr, D., \& Vision, A. (1982). A computational investigation into the human representation and processing of visual information. WH San Francisco: Freeman and Company, 1(2).

Shi, R., Morgan, J. L., \& Allopenna, P. (1998). Phonological and acoustic bases for earliest grammatical category assignment: A cross-linguistic perspective. Journal of child language, 25(1), 169-201.

Shillcock, R. (2014). The Concrete Universal and Cognitive Science. Axiomathes, 24(1), 63-80.

Shillcock, R., Thomas, J., \& Rachael Bailes, R. (submitted). Mirror Neurons, Prediction and Hemispheric Coordination; The Prioritizing of Intersubjectivity over 'Intrasubjectivity'.

Sokolov, E. N., Spinks, J. A., Näätänen, R., \& Lyytinen, H. (2002). The orienting response in information processing. Lawrence Erlbaum Associates Publishers.

Sperber, D., \& Wilson, D. (1995). Relevance: Communication and cognition (2nd. ed.). Oxford, England: Blackwell.

Vygotsky, L. (1934/1986). Thought and language. Cambridge, MA: MIT Press.

Weisberg, M. (2007). Three kinds of idealization. The Journal of Philosophy, 104(12), 639-659. 